\title{
Clinical Profile of Dengue Infection at a Teaching Hospital in South India
}

\author{
Dr Samragni Vasireddy ${ }^{1}$, Dr Aashish Kodali ${ }^{2}$, B Madhu Kiran Reddy ${ }^{2}$, \\ Dr G Eswar ${ }^{3}$ \\ ${ }^{I}$ Dr Samragni Vasireddy, Junior Resident, General Medicine, Dr.Pinnamaneni Siddartha Institute Of Medical \\ Sciences \& Research Foundation, Chinaoutapalli, Krishna Dist, Andhra Pradesh, India. \\ ${ }^{2}$ Dr Aashish Kodali , Junior Resident, General Surgery, Dr.Pinnamaneni Siddhartha Institute Of Medical \\ Sciences \& Research Foundation, Chinaoutapalli, Krishna Dist, Andhra Pradesh, India. \\ ${ }^{2}$ Dr B Madhu Kiran Reddy, Assistant Professor, General Medicine, Dr.Pinnamaneni Siddartha Institute Of \\ Medical Sciences \& Research Foundation, Chinaoutapalli, Krishna Dist, Andhra Pradesh, India. \\ ${ }^{3} \operatorname{Dr}$ G Eswar, Professor, General Medicine, Dr.Pinnamaneni Siddartha Institute Of Medical Sciences \& \\ Research Foundation, Chinaoutapalli, Krishna Dist, Andhra Pradesh, India.
}

\begin{abstract}
:
Background: Dengue fever is the most important viral, mosquito borne infection (Aedes) Dengue virus belongs to the family Flaviviridae. It has become a major epidemic in Indian subcontinent, accounting for nearly one lakh deaths per annum. This is a study on clinical profile of dengue infection.

Method: This is a prospective study of 200 cases admitted in Tertiary Care Hospital. Serum samples were collected from suspected dengue patients and were tested for NSIAg, IgM and IgG antibodies with ELISA kit.

Results: Dengue fever is the most important viral, mosquito borne infection (Aedes) in India. It has become a major epidemic in Indian subcontinent. Spread of the infection is now leading to increased recognition of typical clinical features of dengue infection. Dengue virus belongs to the family Flaviviridae.

Conclusion: Dengue disease continues to involve newer areas, newer populations and is increasing in magnitude, epidemic after epidemic, no vaccine is yet available for protection and the vector control measures are inadequate. Though clinical studies have reported on dengue disease in India, but these are largely based on diagnosis made by kits of doubtful specificity and sensitivity. A lot more remains to be achieved for creating an impact.
\end{abstract}

Keywords: Aedes, Dengue, Flaviridae, NS1Ag

\section{Introduction}

Dengue infection has caused epidemics in india and is a major health problem in our country. WHO estimates that presently about $2 / 5$ ths of the world population is at risk for this viral infection[1]. Dengue was first reported in 1780, when Benjamin Rush described this fever as breakbone fever[2]. Incidence of dengue has increased over 30 fold over the last 50 years.[3]

Risk of mortality I treated cases of DHF/DSS is $1 \%$ while mortality rate among untreated cases escalates to 20\%[4]. India is one of the seven countries in south east asia region regularly reporting incidence of DF/DHF outbreaks due to its high incidence which constantly threteans the health care system.

The $1^{\text {st }}$ confirmed report of dengue infection in india dates back to 1940's and since then more \& more new states have been reporting the disease which mostly strikes in epidemic proportions often inflicting heavy morbidity \& mortality[5]. The common signs and symptoms observed were fever, headache, myalgia, arthralgia and bleeding manifestations have also been observed.

The exact clinical profile is important for patient management and crucial for saving life. The present study is an attempt to describe the salient clinical as well as laboratory findings of serologically confirmed hospitalized cases of dengue fever during the study period[6].

\section{Materials And Methods}

The study was undertaken as a hospital based descriptive study with prospective data collection. Two hundred patients with confirmed dengue fever admitted to tertiary care hospital during a period from January $2014-2016$ were selected for this study.

NS1Ag, IgM and IgG, dengue antibodies were estimated using Rapid strip test. The diagnosis of dengue fever, dengue hemorrhagic feverand dengue shock syndrome was based on the WHOcriteria.

Only those patients were included in the study withclassical features of dengue - fever with chills, bodyache, headache, rash, bleeding manifestations and thrombocytopenia and had a positive strip test. 
Patients who had malaria and enteric fever were excluded from the study. Detailed history and clinicalexaminations were done. A complete blood count, liverfunction tests, renal function tests, chest X-ray and USGabdomen were also done.

\section{Results}

A total of 200 cases admitted to the hospital in Jan 2014 to Jan 2016 were statistically analysed. Most ofdengue cases occurred during the month of August to october depicts the role of rainy season on clusteringof cases.Majority of the case $54 \%$ were male and $46 \%$ were females. Maximum number of cases $61 \%$ was in the age group of $15-40$ years as seen in table 1 .

As seen in table 2 fever was present in all cases and is the most common symptom followed by headache $(90 \%)$, myalgia $(81 \%)$, vomiting $(56 \%)$, abdominal pain $(48 \%)$, breathlessness $(25 \%)$, skin rash $(20 \%)$; haemorrhagic manifestations(25\%) included petechiae, ecchymosis, gum bleeding, haematuria, melena, hematemesis and epistaxis.

Complications have been found in 60 patients $(30 \%)$ which include pleural effusion, hypotension, pneumonia, renal failure, ARDS.Out of 200 cases reviewed patients with anaemia were observed very less. Other laboratory investigations were illustrated in table 4 and 95 patients $(47.5 \%)$ showed haematocrit more than 40,65 patients $(32.5 \%)$ showed leucopenia, thrombocytopenia platelet count less than 1 lakh is seen in 152 patients(76\%), SGOT>40 seen in 66 patients(33\%), SGPT $>40$ seen in 42 patients $(21 \%)$, deranged RFT seen in 5 patients $(2.5 \%)$.

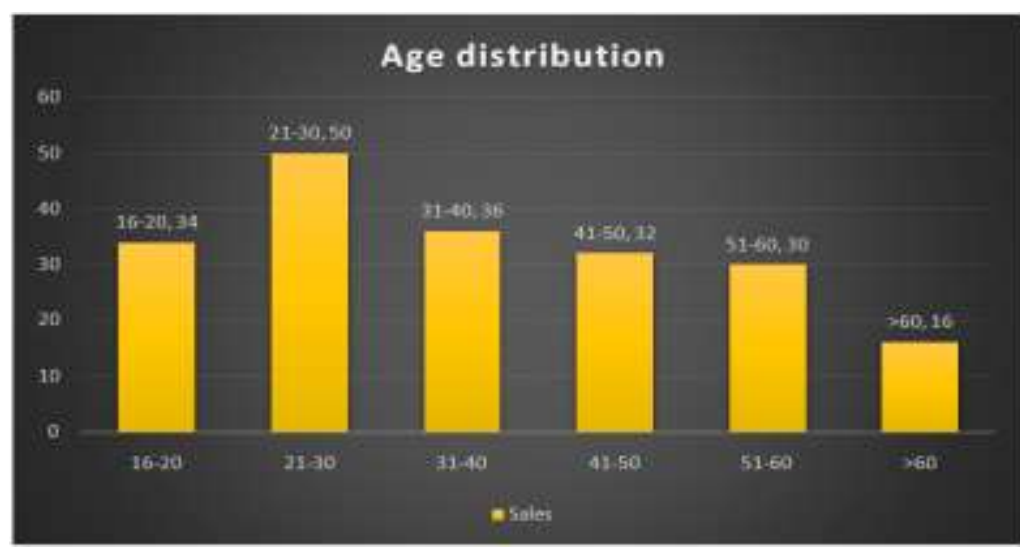

\begin{tabular}{|l|l|}
\hline AGE & NO OF CASES \\
\hline $16-20$ & 34 \\
\hline $21-30$ & 50 \\
\hline $31-40$ & 36 \\
\hline $41-50$ & 32 \\
\hline $51-60$ & 30 \\
\hline$>60$ & 16 \\
\hline
\end{tabular}

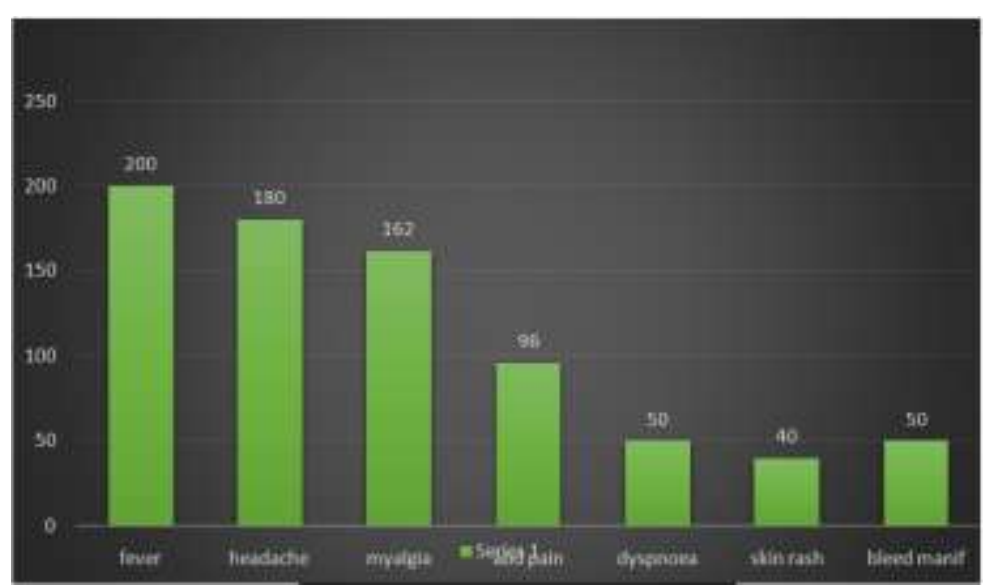




\begin{tabular}{|l|l|}
\hline SYMPTOMS & NUMBER OF CASES \\
\hline Fever & 200 \\
\hline Headache & 180 \\
\hline Myalgia & 162 \\
\hline Abdominal pain & 96 \\
\hline Dyspnoea & 50 \\
\hline Skin rash & 40 \\
\hline Bleeding manifestations & 50 \\
\hline
\end{tabular}

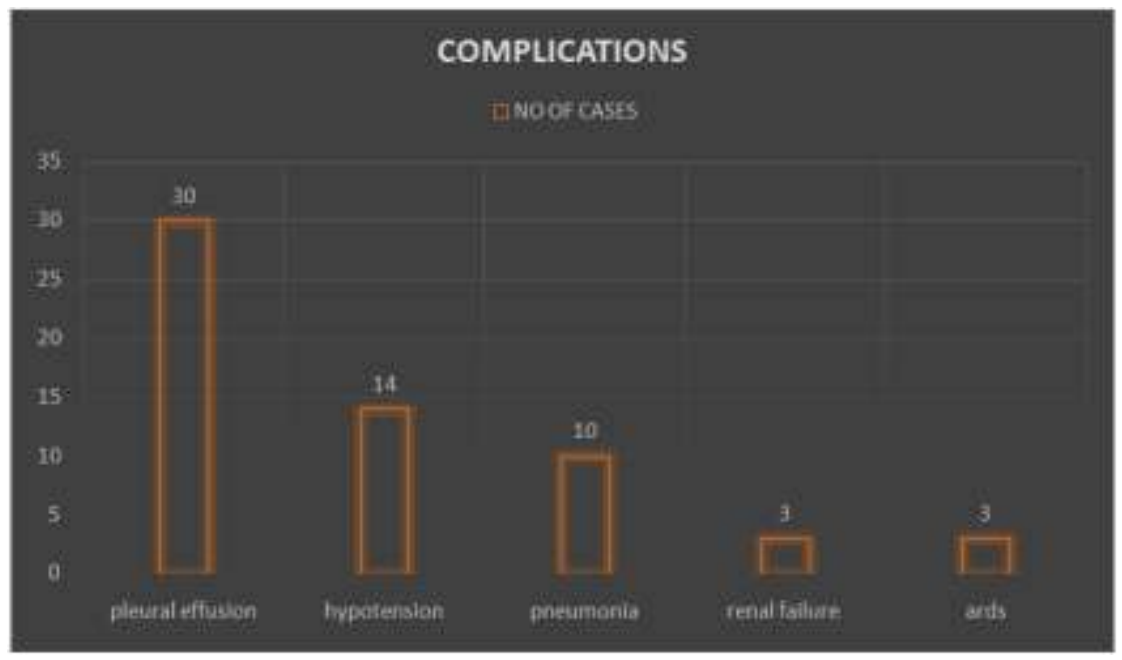

\begin{tabular}{|l|l|}
\hline COMPLICATONS & NO OF CASES \\
\hline Pleural effusion & 30 \\
\hline Hypotension & 14 \\
\hline Pneumonia & 10 \\
\hline Renal failure & 3 \\
\hline ARDS & 3 \\
\hline
\end{tabular}

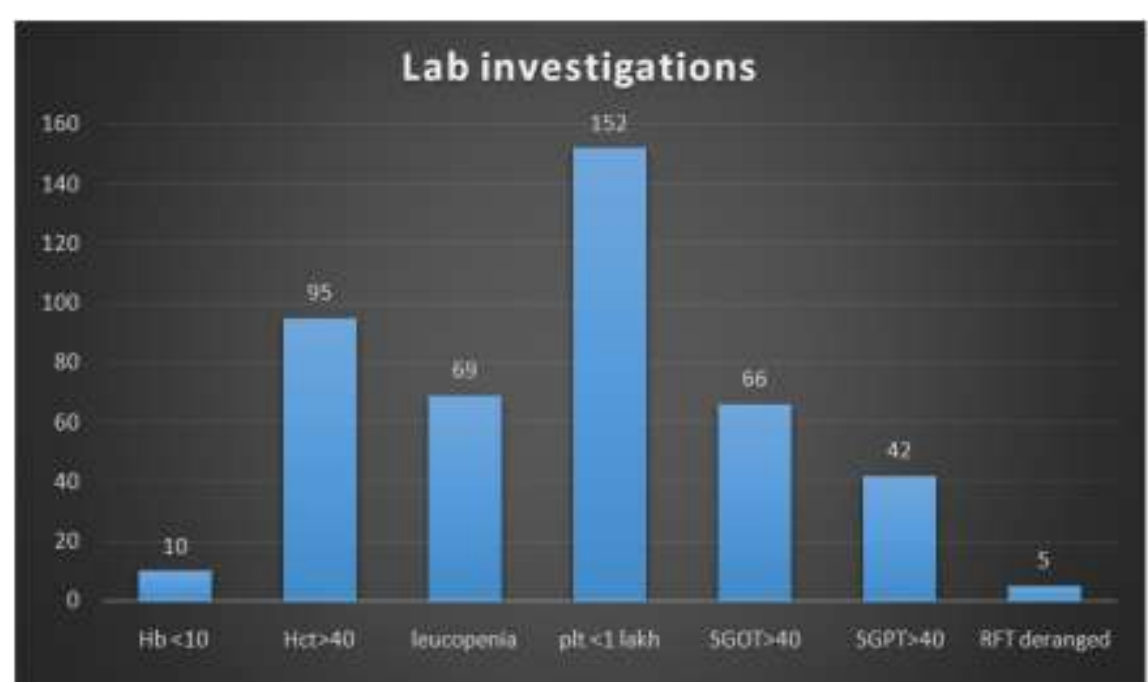

\begin{tabular}{|l|l|}
\hline LAB INVESTIGATIONS & NO OF CASES \\
\hline $\mathrm{Hb}$ & 10 \\
\hline $\mathrm{Hct}$ & 95 \\
\hline Leucopenia & 69 \\
\hline Plt $<$ 1lakh & 152 \\
\hline SGOT $>40$ & 66 \\
\hline SGPT $>40$ & 42 \\
\hline RFT deranged & 5 \\
\hline
\end{tabular}




\section{Discussion}

Dengue is an important emerging disease of the tropical and sub-tropical regions today. Since the first confirmed case of dengue in India, during the late 1940s[7]. In the present study maximum number of patients were admitted in the rainy season (August to October) that is related to favourable conditions for growth of vector Aedes aegypti[8]. [9]Transmission of dengue increases during monsoon. [9]the correlation between occurrence of dengue and monsoon is clearly evident in this study and previous studies conducted.[7]In the present study maximum number of patients who suffered were in the age group between 15-40 years, Our findings were related with Doke et al, as maximum number of patients occurred in age group 15-40 years.[10]The male to female ratio is found to $1.7: 1$, the study conducted by ashwini kumar et al reveals ratio 1.17:1.[7]where as another study showed slightly similarity in ratio was $1.3: 1$ by anagha $G$ kinikar et al.[11]Almost all the studies had male preponderance among affected individuals.

The clinical profile of dengue shows that fever was the most common presenting symptom in 200 (100\%) patients. Abdominal symptoms/signs such as abdominal pain, nausea/vomiting, anorexia, abdominal tenderness were found to be present $48 \%$ of study population which shows identical result statistically where as another study was conducted by Satya sudhish Nimmagadda which shows less number of patients are affected with abdominal symptoms.

[12]Mavilla anuradha et al, shows frequently affected symptoms in their study population are myalgia followed by headache, vomiting etc. which are reported similar frequency of all symptoms related to our study.[13]

Bleeding manifestations were revealed in $25 \%$ of patients while Ashwini Kumar et al, reported in $26.6 \%[7]$ and Tejashree .A et al, were reported in 3.84\% of patients.[14]

Our study shows pleural effusion was found in $15 \%$ patients where other study displayed ARDS (33.33\%) as a significant complication[7]but our study revealed that ARDS was found to be least.Other complications such as renal failure each was observed in $1.5 \%$ patients in our study whereas other study shows renal failure was found in 40.6\%.In our study, Hypotension was observed in 14 (7\%) of patients but no death was found whereas other study was reported 3 deaths due to hypotension in seropositive patients.[12]

A similar study was conducted by Ashwini kumar et al, shows statistically significant result as our study in complications of pneumonia, renal failure [7]. The laboratory investigations are evaluated in our study, the finding shows that anemia was associated in least patients compare to other study was conducted by Tejushree .A et al. which shows significant difference in both study.[14] Increased hematocrit was observed in $47.5 \%$ of patients whereas Mavilla Anuradha et al, were reported in $30.00 \%$ of patients. $152(76 \%)$ patients had platelet count $<100000$ cells/cumm but Rashmi K.S et al reported $72.77 \%$ of patients had platelet count $<$ 100000 cells/cumm. Soor study reflected that more patients are encountered with thrombocytopenia[15]. Leukopenia was observed in $32.5 \%$ of patients whereas prafulla dutta et al, were reported $30 \%$ of patients presented with leukopenia.[16]

Liver enzymes like AST was found in 1/4th of study population whereas Prafulla Dutta et al, were reported in 1/3rd of study population[16] and ALT were in 1/4th of study population whereas other study shows half of the patients.[8]

[12]The various factors were responsible for thrombocytopenia such as platelet dysfunction, consumption coagulopathy and endothelial dysfunction which are not related to severity of bleeding. The patients were also investigated for other causes of fever endemic in our region such as malaria, typhoid and leptospirosis which causes the thrombocytopenia and often lead to delay in diagnosis of dengue. No deaths were found in our prospective study.

\section{Conclusion}

Dengue disease continues to involve newer areas, newer populations and is increasing in magnitude, epidemic after epidemic, no vaccine is yet available for protection and the vector control measures are inadequate. Though clinical studies have reported on dengue disease in India, but these are largely based on diagnosis made by kits of doubtful specificity and sensitivity. A lot more remains to be achieved for creating an impact.

\section{References}

[1]. World Health Organization; Dengue and Dengue Hemorrhagic fever. Available in www.who.int/media centre/factsheets./ fs117/en/accessed on 19.4.2013

[2]. Guzmán MG, Kourí G; Dengue: Anupdate. Lancet Infect Dis., 2002; 2: 33-42.

[3]. WHO ; Dengue - World Health Organizationwww.who.int/denguecontrol/en

[4]. World health Organization. Dengue anddengue haemorrhagic fever. Fact Sheet.No.117, 2002. Availablefrom:http//www.who.int/mediacentre/factsheets/fs117/en/

[5]. Dengue in Kerala: A critical review. ICMRBulletin. 2006;36:13-22.

[6]. Konar NR, Mandal AK, Saha AK.Hemorrhagic fever in Kolkata. J AssocPhysicians India. 1966;14:331-40. 
[7]. Ashwini K, Chythra RR, Vinay P, Seema S, Channuveerappa B, Charmaine MS. Clinical manifestations and trend of dengue cases admitted in a tertiary care hospital, udupi district, Karnataka. Indian Journal of Community Medicine 2010; 35(3): 386-390

[8]. Mavilla A, Rahul HD. Screening and manifestations of seropositive dengue fever patients in perambalur: A hospital based study. International journal of Medical Science and Public Health 2014; 3(6): 745 - 748

[9]. Gunusekaran P, Kaveri K, Mohana S, Kavita A, Sureshbabu BV, Padmapriya P, Kiruba R, et al. Dengue disease status in Chennai (2006-2008): A Retrospective analysis. Indian J Med Res 2011; 133: 322-325

[10]. Doke P, Pawar S. Profile of Dengue fever outbreaks in Maharashtra. Indian J Community Med 2000; 25:170-6.

[11]. Saini S, Anagha GK, Sachin D, Deepika B, Roushni SB. Epidemiology and Seropositivity of dengue cases in a rural tertiary care hospital of western Maharashtra, India. IJBAR 2013; 4(7): 473-77.

[12]. Satya SN, Chakrapani M, Archit B, Pavan MR, Akshatha NU. Atypical manifestations of Dengue Fever (DF) - Where do we stand today?. J Clin Diag Res 2014; 8(1): 71-73.

[13]. Neeraja M, Lakshmi V, Teja VD, Umabala P, Subbalakshmi MV. Serodiagnosis of dengue virusinfection in patients presenting to a tertiary care hospital. Indian J Med Microbiol 2006; 24(4): 280- 282.

[14]. Tejushree A, Thejaswini HS, Madhuri K. A serological study of Dengue and Hanta virus in acute febrile patients in a tertiary care hospital. International Journal of Pharmaceutical Science Invention 2014; 3(7): 22-25.

[15]. Rashmi KS, Jagdeesh, Ravikumar KL, Pratibha MJ, Giridhar UP, Arun KB. Serological markers prevalence and trend of probable dengue infection at a tertiary care hospital in Banglore. Journal of Evolution of Medical and Dental Sciences 2013; 12(36): 69686976.

[16]. Prafulla D, Siraj AK, Jani B, Jagadish M. Demographic and clinical features of patients withdengue in Northeastern region of India: A Retrospective cross-sectional study during 2009- 2011. Journal of virology and Microbiology 2012; 2012: 1-11. 